\title{
HERMITIAN FORMS AND THE FIBRATION OF SPHERES
}

\author{
PAUL BINDING
}

\begin{abstract}
We identify the real $(2 n-1)$-dimensional sphere $S^{2 n-1}$ with the unit sphere of $\mathbf{F}^{2}$, where $\mathbf{F}=$ reals, complexes or quaternions and $n=1,2$ or 4 , respectively. It is shown how any Hermitian form over $\mathbf{F}^{2}$, restricted to $S^{2 n-1}$, is related to the (double covering for $n=1$, Hopf for $n=2,4$ ) fibration

$$
\left(x_{1}, x_{2}\right) \rightarrow\left(\left|x_{1}\right|^{2}-\left|x_{2}\right|^{2}, 2 x_{1} \bar{x}_{2}\right): S^{2 n-1} \rightarrow S^{n} .
$$

Convexity of the joint range of several Hermitian forms over the unit sphere of an arbitrary normed vector space $V$ over $\mathbf{F}$, with $\operatorname{dim} V>2$, is deduced as a corollary.
\end{abstract}

1. Introduction. We shall present a connection between an easily constructed basis for the Hermitian forms over $\mathbf{F}^{2}$ and the "components" of a standard fibration of the $(2 n-1)$-dimensional sphere. Here $\mathbf{F}$ is either the real field $\mathbf{R}(n=1)$, the complex field $\mathbf{C}(n=2)$ or the quaternion skew field $\mathbf{R}(n=4)$.

By a Hermitian form $\phi$ over a normed (left) vector space over $\mathbf{F}$ we mean a sesquilinear form evaluated on the diagonal. Thus $\phi(x)=f(x, x)$, where $f: V \times V \rightarrow$ $\mathbf{F}, f(x, y)$ is linear in $x$ and $\overline{f(x, y)}=f(y, x)$. An easy computation shows that, in the case $V=\mathbf{F}^{2}, x=\left(x_{1}, x_{2}\right)$, we may take

$$
\phi(x)=\left[x_{1} x_{2}\right]\left[\begin{array}{ll}
a & \bar{c} \\
c & b
\end{array}\right]\left[\begin{array}{l}
\bar{x}_{1} \\
\bar{x}_{2}
\end{array}\right]=a\left|x_{1}\right|^{2}+b\left|x_{2}\right|^{2}+2 \operatorname{Re}\left(x_{1} \overline{c x}_{2}\right)
$$

where $(a, b, c) \in \mathbf{R}^{2} \times \mathbf{F}$. (Details of these constructions are given for $\mathbf{F}=\mathbf{H}$ in $\S 2$.) There is an obvious inner product space isomorphism between $\mathbf{F}$ and $n$-dimensional real Euclidean space $\mathbf{E}^{n}$, so the forms (1) generate an $\mathbf{E}^{n+2}$.

In homogeneous form, the fibration in question has the formula

$$
\Psi: x \rightarrow\left(\left|x_{1}\right|^{2}+\left|x_{2}\right|^{2},\left|x_{1}\right|^{2}-\left|x_{2}\right|^{2}, 2 x_{1} \bar{x}_{2}\right): \mathbf{F}^{2} \rightarrow \mathbf{R}^{2} \times \mathbf{F} .
$$

Again identifying $\mathbf{R}^{2} \times \mathbf{F}$ with $\mathbf{E}^{n+2}$, we shall show in $\S 2$ that the components $\left(\psi_{1}, \ldots, \psi_{n+2}\right)$ of $\boldsymbol{\Psi}$ form an orthogonal basis for the space of Hermitian forms. Restricted to the unit sphere $\psi_{1}^{-1}(1)$ of $\mathbf{F}^{2}$, the remaining components $\hat{\Psi}=\left(\psi_{2}, \ldots\right.$, $\left.\psi_{n+2}\right)$ decompose $S^{2 n-1}$ into fibres $S^{n-1}$ over a base space $S^{n}$. In particular, $\hat{\Psi}$ is not homotopic to a constant map. In $\S 3$ we use this to show that the "joint range" $\Phi(U)$ is convex when $\Phi=\left(\phi_{1}, \ldots, \phi_{m}\right)$ is an $m$-tuple of Hermitian forms, $m \leq n+1, U$ is the unit sphere of $V$ and $\operatorname{dim} V>2$. $\S 2$ covers the case $\operatorname{dim} V=2$, at least up to affine equivalence, as we shall see.

Some applications of, and related work on, these matters form the subject of a forthcoming survey by the author, but the following may be noted here. The convexity result is due to Brickman [4] when $\mathbf{F}=\mathbf{R}$ and implies the ToeplitzHausdorff theorem (see [7]) when $\mathbf{F}=\mathbf{C}$. Our methods are related to those of

Received by the editors April 9, 1984.

1980 Mathematics Subject Classification. Primary 15A63, 55R25.

(c)1985 American Mathematical Society $0002-9939 / 85 \$ 1.00+\$ .25$ per page 
McIntosh [7] for $\mathbf{F}=\mathbf{R}$ and Davis [5] and Atkinson [1] for $\mathbf{F}=\mathbf{C}$, but the connection between (1) and (2) is more explicit here. Au-Yeung and Poon [2] have recently shown for $\mathbf{F}=\mathbf{C}$ (and stated for $\mathbf{F}=\mathbf{H}$ ) that the convexity result is equivalent to earlier theorems of Bohnenblust [3] and Friedland and Loewy [6], but this route involves completely different arguments and is somewhat indirect.

ACKNOWLEDGement. I am most grateful to Professor Atkinson for a copy of his notes [1] on the convexity of $\Phi(V \backslash\{0\})$ for $\mathbf{F}=\mathbf{C}, m=3$ which were the starting point for my investigation.

2. Forms and fibrations. The simplest case is $\mathbf{F}=\mathbf{R}$. Then the choices

$$
(a, b, c)=(1,1,0),(1,-1,0) \text { and }(0,0,1)
$$

form an orthogonal basis of the real Euclidean space $\mathbf{E}^{3}$. In terms of (1), we have a basis for the Hermitian (i.e. symmetric) forms on $\mathbf{R}^{2}$. The second and third forms generate

$$
\hat{\Psi}: x \rightarrow\left(x_{1}^{2}-x_{2}^{2}, 2 x_{1} x_{2}\right): \mathbf{R}^{2} \rightarrow \mathbf{R}^{2}
$$

as in (2). Restricted to $\psi_{1}^{-1}(1)=\left\{x: x_{1}^{2}+x_{2}^{2}=1\right\}$, $\hat{\Psi}$ provides a double covering of $S^{1}$. This is a consequence of the general machinery below $[10, \S 20.4]$ but can also be seen from the complex version. Indeed if we write ${ }^{\mathbf{C}} y=y_{1}+i y_{2}$ for $y \in \mathbf{R}^{2}$ then ${ }^{\mathbf{C}} \hat{\Psi}(x)=\left({ }^{\mathbf{C}} x\right)^{2}$.

The next case is $\mathbf{F}=\mathbf{C}$, which is inner product isomorphic to $\mathbf{E}^{2}$ via

$$
\left\langle{ }^{\mathbf{C}} x,{ }^{\mathbf{C}} y\right\rangle=x_{1} y_{1}+x_{2} y_{2} .
$$

If $z={ }^{\mathbf{C}} y$ then we write $y={ }^{\mathbf{R}} z$. Appending $(a, b, c)=(0,0, i)$ to (3), we obtain

$$
\left(a, b,{ }^{\mathbf{R}} c\right)=(1,1,0,0),(1,-1,0,0),(0,0,1,0) \text { and }(0,0,0,1)
$$

as an orthogonal basis of $\mathbf{E}^{4}$ representing the Hermitian forms on $\mathbf{C}^{2}$. From (1), the final three forms generate a mapping

$$
z \rightarrow\left(\left|z_{1}\right|^{2}-\left|z_{2}\right|^{2}, 2 \operatorname{Re}\left(z_{1} \bar{z}_{2}\right), 2 \operatorname{Im}\left(z_{1} \bar{z}_{2}\right)\right): \mathbf{C}^{2} \rightarrow \mathbf{R}^{3},
$$

and the final two components are just ${ }^{\mathbf{R}}\left(2 z_{1} \bar{z}_{2}\right)$. Comparing this with (2), we have the real components of a mapping $\hat{\Psi}$ which, restricted to $\psi_{1}^{-1}(1)=\left\{z:\left|z_{1}\right|^{2}+\left|z_{2}\right|^{2}=\right.$ $1\}$, is the Hopf fibration: $S^{3} \rightarrow S^{2}$, essentially in the form given by Milnor $[\mathbf{9}, \mathrm{p}$. 102].

We need some preparation for the case $\mathbf{F}=\mathbf{H}$. A quaternion $q$ may be represented in the form

$$
q=c+j d, \quad(c, d) \in \mathbf{C}^{2},
$$

where $j^{2}=-1$ and $d j=j \bar{d}$. We write $\bar{q}=\bar{c}-j d,|q|^{2}=q \bar{q}, 2 \operatorname{Re} q=q+\bar{q}$ and $\alpha(q)=c+d j$. Evidently, $\alpha(\bar{q})=\overline{\alpha(q)}$ so

$$
|\alpha(q)|=|q| \quad \text { and } \quad \alpha^{2}(q)=q .
$$

We also write

$$
{ }^{\mathbf{R}} q=\left({ }^{\mathbf{R}} c,{ }^{\mathbf{R}} d\right) \in \mathbf{R}^{4} .
$$

This makes $\mathbf{H}$ isomorphic (in an obvious inner product) to $\mathbf{E}^{4}$, with $|q|=\left\|{ }^{\mathbf{R}} q\right\|$. 
Appending $(a, b, c)=(0,0, j)$ and $(0,0, j i)$ to the choices (4) for $\mathbf{C}$, we obtain an orthogonal basis of elements $\left(a, b,{ }^{\mathbf{R}} c\right)$ for $\mathbf{E}^{6}$ representing the Hermitian forms on $\mathbf{H}^{2}$. The final five forms (1) generate a mapping

$$
\begin{aligned}
& \Omega: \mathbf{H}^{2} \rightarrow \mathbf{R}^{5}:\left(q_{1}, q_{2}\right)=\left(c_{1}+j d_{1}, c_{2}+j d_{2}\right) \\
& \rightarrow\left(\left|q_{1}\right|^{2}-\left|q_{2}\right|^{2}, 2 \operatorname{Re}\left(c_{1} \bar{c}_{2}+\bar{d}_{1} d_{2}\right),\right. \\
&\left.\quad 2 \operatorname{Im}\left(c_{1} \bar{c}_{2}-\bar{d}_{1} d_{2}\right), 2 \operatorname{Re}\left(\bar{c}_{2} \bar{d}_{1}-c_{1} d_{2}\right), 2 \operatorname{Im}\left(c_{1} d_{2}+\bar{c}_{2} \bar{d}_{1}\right)\right) .
\end{aligned}
$$

The final four components of $\Omega\left(q_{1}, q_{2}\right)$ equal ${ }^{\mathbf{R}} p$ where

$$
p=2\left(c_{1} \bar{c}_{2}+d_{1} \bar{d}_{2}\right)+2 j\left(\bar{c}_{2} \bar{d}_{1}-\bar{c}_{1} \bar{d}_{2}\right)=2 \alpha\left(q_{1}\right) \overline{\alpha\left(q_{2}\right)} .
$$

Thus $\Omega$ furnishes the real components of $\hat{\Psi} \circ \alpha, \hat{\Psi}$ as in (2). We have almost established the following

THEOREM 1. Let $n$ be the dimension of $\mathbf{F}$, considered as a real vector space. Then a basis $\psi_{1}, \ldots, \psi_{n+2}$ for the Hermitian forms over $\mathbf{F}^{2}$ exists which, restricted to the unit sphere, has the following properties: (i) $\psi_{1}$ has constant value 1, (ii) $\hat{\Psi}=\left(\psi_{2}, \ldots, \psi_{n+2}\right)$ provides a fibration of $S^{2 n-1}$ with base space $S^{n}$ and fibres $S^{n-1}$.

To complete the argument for $\mathbf{F}=\mathbf{H}$, we denote the one-point compactification of $\mathbf{H}$ by $\mathbf{H} \cup\{\infty\}$, and, defining $\alpha(\infty)=\infty$, we readily verify via (5) that $\alpha$ is a homeomorphism of $\mathbf{H} \cup\{\infty\}$ onto itself. This allows the argument of [8, p. 67 or 10, p. 109] for the Hopf map to go through for $\hat{\Psi} \circ \alpha$-again we are identifying the restriction of $\hat{\Psi}$ to $\psi_{1}^{-1}(1)=\left\{q:\left|q_{1}\right|^{2}+\left|q_{2}\right|^{2}=1\right\}$ with the Hopf map $S^{7} \rightarrow S^{4}$ (cf. $[9$, p. 102]).

3. Convexity of joint ranges. As in the introduction, let $V$ be a normed vector space over $\mathbf{F}$, with $U=\{x \in V:\|x\|=1\}$, and let $\Phi=\left(\phi_{1}, \ldots, \phi_{m}\right)$ be an $m$-tuple of Hermitian forms on $V$. For notational convenience we view $\alpha$ as the identity on $\mathbf{R}$ and $\mathbf{C}$. This section is devoted to the following result:

THEOREM 2. (i) If $\operatorname{dim} V=1$ then $\Phi(U)$ is a point.

(ii) If $\operatorname{dim} V=2$ then $\Phi(U)$ is an affine image of $S^{n}$ and is thus either a (convex) affine disc or a (nonconvex) affine sphere. In the latter case, $m \geq n+1$.

(iii) If $\operatorname{dim} V>2$ (e.g. $\operatorname{dim} V$ is infinite) and $m \leq n+1$ then $\Phi(U)$ is convex.

PROOF. (i) follows from the representation $\phi_{j}(x)=a_{j}|x|^{2}-$ cf. (1) with $x_{2}=0$.

(ii) follows mostly from Theorem 1. Evidently $\left.\phi_{j}\right|_{U}$ is an affine combination of the real components of $\hat{\Psi} \circ \alpha$ which maps onto $S^{n}$. The final contention follows from the fact that $m<n+1$ forces the affine map $\Phi(U) \rightarrow S^{n}$ to be singular.

(iii) By appending zero forms if necessary, we may assume without loss of generality that $m=n+1$.

Suppose $\Phi(U)$ is not convex. Then there are $u_{j} \in U$ and a point $b$ on the line segment joining $\Phi\left(u_{1}\right)$ to $\Phi\left(u_{2}\right)$ such that

$$
b \notin \Phi(U) .
$$

Let $T$ be the span of $u_{1}$ and $u_{2}$. By (6), $\Phi(U \cap T)$ is nonconvex, so by (i) and (ii), $\operatorname{dim} T=2$. By Theorem 1 , an affine isomorphism exists under which $\left.\Phi\right|_{T}$ becomes $\hat{\Psi} \circ \alpha$. For notational simplicity we shall therefore assume $\left.\Phi\right|_{T}=\hat{\Psi} \circ \alpha$. 
Let $u_{3} \in U \backslash T$ and let $W$ be the set of elements $w=\sum_{j=1}^{3} a_{j} u_{j} \in U$ such that $a_{3}$ is nonnegative real. Since $T \cap U$ is an $S^{2 n-1}, W$ is homeomorphic to a hemisphere bounded by $S^{2 n-1}$, i.e. to a real $2 n$-dimensional ball $B^{2 n}$. Thus $\left.\Phi\right|_{W}$ is a continuous extension of $\hat{\Psi} \circ \alpha$ from $S^{2 n-1}$ to $B^{2 n}$. In particular, $\hat{\Psi} \circ \alpha$ is homotopic to a constant, and this is a contradiction [8, p. 67, Proposition 5.1].

\section{REFERENCES}

1. F. V. Atkinson, On the range of a set of three Hermitian quadratic forms, unpublished, 1981.

2. Y.-H. Au-Yeung and Y.-T. Poon, A remark on the convexity and positive definiteness concerning Hermitian matrices, Southeast Asian Bull. Math. 3 (1979), 85-92.

3. F. Bohnenblust, Joint positiveness of matrices, unpublished.

4. L. Brickman, On the field of values of a matrix, Proc. Amer. Math. Soc. 12 (1961), 61-66.

5. C. Davis, The shell of a Hilbert-space operator. II, Acta Sci. Math. (Szeged) 31 (1970), 301-318.

6. S. Friedman and R. Loewy, Subspaces of symmetric matrices containing matrices with a multiple first eigenvalue, Pacific J. Math. 62 (1976), 389-399.

7. A. McIntosh, The Toeplitz-Hausdorff theorem and ellipticity conditions, Amer. Math. Monthly 85 (1978), 475-477.

8. S.-T. Hu, Homotopy theory, Academic Press, New York, 1959.

9. J. Milnor, Singular points of complex hypersurfaces, Princeton Univ. Press, Princeton, N. J., 1968.

10. N. Steenrood, The topology of fibre bundles, Princeton Univ. Press, Princeton, N. J., 1951.

Department of MATHEMATiCs AND Statistics, UNiversity of Calgary, CALGARY, ALBERTA, CANADA T2N 1N4 